\title{
The magnetic and magnetoresistance properties of ultrathin magnetite films grown on MgO substrate
}

\author{
Y. Zhou, ${ }^{\text {a) }}$ Ciaran McEvoy, R. Ramos, and I. V. Shvets \\ SFI Nanoscience Laboratories, School of Physics, Trinity College, Dublin 2, Ireland
}

(Presented on 3 November 2005; published online 26 April 2006)

\begin{abstract}
We report on the properties of ultrathin $(2,4,6$, and $8 \mathrm{~nm})$ epitaxial films of magnetite, $\mathrm{Fe}_{3} \mathrm{O}_{4}$, grown on $\mathrm{MgO}$ (100). Atomic force microscopy image and $V-I$ curves suggest that the films at this thickness are still continuous. The resistivity versus temperature results imply that the conductivity mechanism in all these films is similar. The resistivity of $4 \mathrm{~nm}$ thick film is much greater than that of 6 and $8 \mathrm{~nm}$ films. The films show ferrimagnetic instead of reported superparamagnetic behavior. The dead layer formed by $\mathrm{Mg}$ diffusion between $\mathrm{MgO}$ substrate and magnetite films and also the dead layer on the top uncapped film could be the possible reasons for the anomalous resistivity and magnetic properties of the ultrathin films. The effect of the "dead layer" in the thinner film is relatively greater than the one in the thicker film and should lead to a lower magnetoresistance. (c) 2006 American Institute of Physics. [DOI: 10.1063/1.2176313]
\end{abstract}

\section{INTRODUCTION}

Magnetite, $\mathrm{Fe}_{3} \mathrm{O}_{4}$, is a well-known ferrimagnetic spinel since it is both conducting and magnetic with a high Curie temperature of $858 \mathrm{~K}$. Band structure calculations indicate a half-metallic structure with a gap in the density of states of majority carriers. ${ }^{1,2}$ These properties make magnetite a very attractive material for applications in various spin-electronic devices. Epitaxial $\mathrm{Fe}_{3} \mathrm{O}_{4}$ films grown on $\mathrm{MgO}$ substrates are known to contain antiphase domain boundaries (APBs). ${ }^{3}$ The $\mathrm{APBs}$ are natural growth defects, resulting from the lattice constant of $\mathrm{Fe}_{3} \mathrm{O}_{4}$ being twice as large as the one of $\mathrm{MgO}$ or from the rotational symmetry of the film being lower than that of the substrate. The effects of APBs on the magnetic, resistivity, and magnetotransport properties were intensively investigated. It was reported that the magnetization does not saturate in high field, ${ }^{3}$ and ultrathin films below $5 \mathrm{~nm}$ show superparamagnetic behaviour. ${ }^{4}$ The APBs are also known to increase the resistivity of epitaxial $\mathrm{Fe}_{3} \mathrm{O}_{4}$ films as they lead to additional scattering of conduction electrons. ${ }^{5}$ Eerenstein et al. ${ }^{5}$ reported magnetoresistance (MR) behavior of ultrathin magnetite films with thickness greater than $6 \mathrm{~nm}$ as spinpolarized transport across antiferromagnetic interfaces. In this paper we systematically investigated the magnetic, resistivity, and magnetoresistance (MR) properties, and topography of ultrathin magnetite films with a thickness of $2-8 \mathrm{~nm}$.

\section{EXPERIMENTS}

In this study we used epitaxial magnetite films with thicknesses of 2, 4, 6, and $8 \mathrm{~nm}$ grown on $\mathrm{MgO}$ (001) single crystal substrates. The films were grown by oxygen-plasmaassisted molecular beam epitaxy (MBE). The substrate temperature during the growth was $250^{\circ} \mathrm{C}$. Other details of the film growth are presented in Ref. 6. Growth mode and crystalline quality of the films were monitored in situ by reflection high-energy electron diffraction (RHEED). The film thickness was controlled by quartz-crystal thickness moni-

${ }^{a)}$ Electronic mail: zhouy@tcd.ie tors calibrated by $\mathrm{x}$-ray reflectivity. Raman spectroscopy (RS) measurements were carried out to further confirm that the films were magnetite. The four-probe method was employed for the resistance and MR measurements. The external magnetic field was applied in the film plane and parallel to (100) direction, the direction of the current $(1 \mu \mathrm{A}$ dc current). MR is defined as $\left(R_{H}-R_{0}\right) / R_{0}$, where $R_{H}$ and $R_{0}$ denote the resistance in the presence and absence of the applied external field, respectively. Hysteresis loop and magnetic moment measurements were carried out using a MicroMag 3900 alternating gradient force magnetometer (AGFM) at room temperature. Commercial $\mathrm{Si}$ cantilevers were employed for atomic force microscopy (AFM). The microscope tip was scanning in semicontact mode for surface morphology.

\section{RESULTS AND DISCUSSION}

The RHEED results suggest that the magnetite films were grown epitaxially as confirmed by the oscillations of pattern intensity. AFM technique was employed to study the surface topography of magnetite films. As shown in Fig. 1, the $2 \mathrm{~nm}$ thick film was not granular but rather continuous.

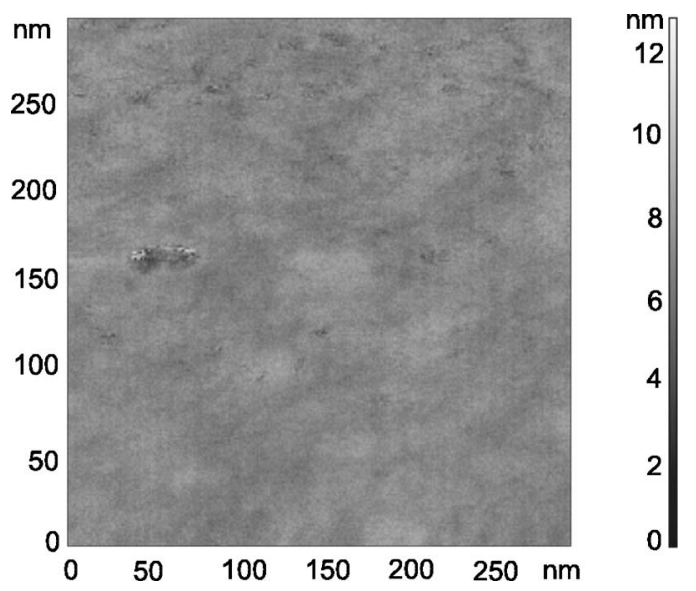

FIG. 1. AFM image of the $2 \mathrm{~nm}$ thick $\mathrm{Fe}_{3} \mathrm{O}_{4}$ film. 


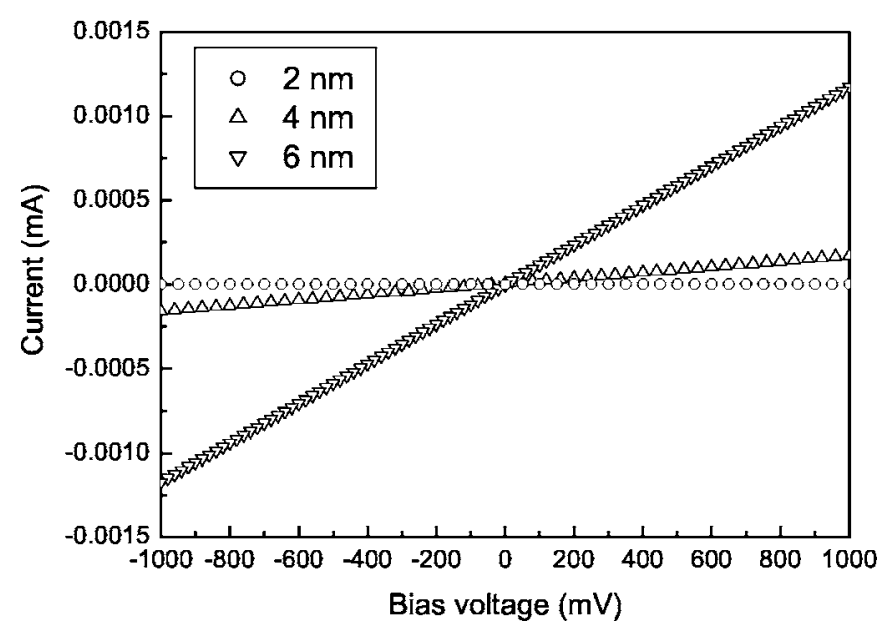

FIG. 2. $V$-I curves of the 2, 4, and $6 \mathrm{~nm}$ thick magnetite films.

The average roughness is $0.5 \mathrm{~nm}$ over $300 \times 300 \mathrm{~nm}$ area which indicates a high surface quality of the films. The $V-I$ curve was used to further investigate if the film was continuous. A linear $V-I$ curve is expected to indicate continuous film and the discontinuous films should show substantially nonlinear $V$ - $I$ curve. Figure 2 shows the $V-I$ curves of the 2 , 4 and $6 \mathrm{~nm}$ thick magnetite films. The linearity of the curves suggests that the film is continuous even with a thickness of $2 \mathrm{~nm}$.

The resistivities of the 4,6 , and $8 \mathrm{~nm}$ thick magnetite films with temperature were shown in Fig. 3. The resistivity of the $4 \mathrm{~nm}$ thick film is much higher than that of the 6 and $8 \mathrm{~nm}$ thick films. We do not observe a jumplike behavior in the resistivity around $100-120 \mathrm{~K}$ temperature range corresponding to the Verwey transition. The absence of Verwey transitions in thin films has already been reported. ${ }^{7-9}$ Mott $^{8}$ explained it by blocking of the $\mathrm{Fe}^{2+} / \mathrm{Fe}^{3+}$ fluctuations by impurities. Garcia et al. ${ }^{9}$ discussed the origin of the Verwey transition related to the oxygen sites condensation. The activation energies $U$ were determined from a fit of a thermally activated resistivity, $\rho=\rho_{a} \exp (-U / k T)$. The deduced data were given in Table I. Whereas the high-temperature values are in agreement with the values reported by Feng et al. ${ }^{10}$ the low-temperature values are seen to be lower than the bulk

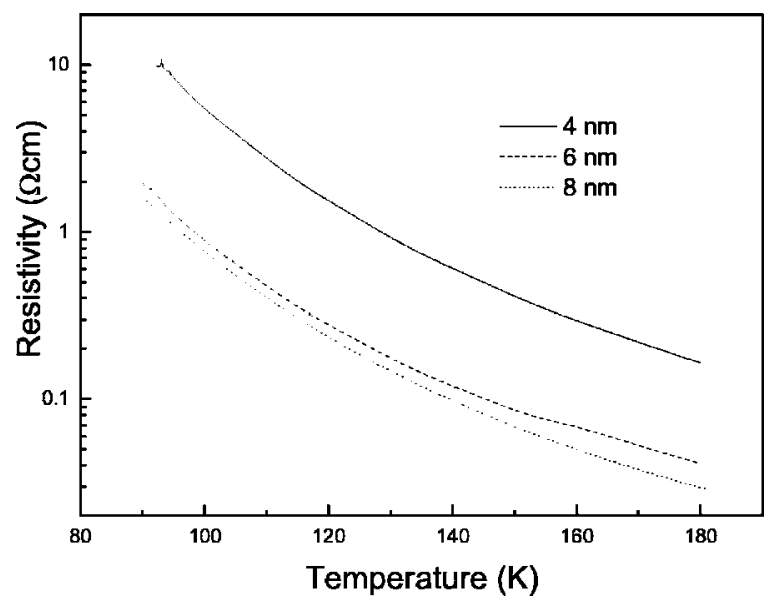

FIG. 3. The resistivity of the 4,6 , and $8 \mathrm{~nm}$ magnetite films vs temperature.
TABLE I. Activation energies determined above and below $100 \mathrm{~K}$ for the samples.

\begin{tabular}{ccc}
\hline \hline & \multicolumn{2}{c}{$U(\mathrm{meV})$} \\
\cline { 2 - 3 } $\begin{array}{c}\text { Film thickness } \\
(\mathrm{nm})\end{array}$ & $T>100 \mathrm{~K}$ & $T<100 \mathrm{~K}$ \\
\hline 4 & 67.8 & 68.9 \\
6 & 68.6 & 71.7 \\
8 & 64.1 & 67 \\
\hline \hline
\end{tabular}

one $(105 \mathrm{meV})$. All the activated energy values of the ultrathin films are similar. This implies that the conductivity mechanism in these films is the same. This result is in line with the conclusion from the $V$-I curve study.

The low field hysteresis loop of $4 \mathrm{~nm}$ ultrathin magnetite film was shown in Fig. 4. The diamagnetic ( $\mathrm{MgO}$ and holder) contributions were subtracted from the measured moments. The saturation magnetization increases with the film thickness. The values of saturation magnetization of the 4,6 , and $8 \mathrm{~nm}$ thick films are 303,371 , and $380 \mathrm{emu} / \mathrm{cm}^{3}$ which is lower than the one of magnetite film with a thickness of $100 \mathrm{~nm}\left(390 \mathrm{emu} / \mathrm{cm}^{3}\right)$. The result is in contradiction with the reports that films below $5 \mathrm{~nm}$ are superparamagnetic. ${ }^{4}$ The loop is unsaturated because of the antiferromagnetical coupling through APBs. The coercivity increases with the film thickness. The coercivities for 4 and $6 \mathrm{~nm}$ magnetites are 4.6 and 10 Oe, respectively, which are much lower than the values for the thicker films.

We already reported that in the thickness range of up to $100 \mathrm{~nm}$ the as-grown films as well as the annealed ones are fully strained on the $\mathrm{MgO}$ substrate. ${ }^{6,11}$ Therefore, a change in the strain state does not appear to be responsible for the anomaly properties as all the films are equally strained. The dead layer formed by $\mathrm{Mg}$ diffusion between the $\mathrm{MgO}$ substrate and the magnetite films and also the dead layer on the top uncapped film are considered as the possible reasons for the anomalies in resistivity and magnetic properties of the ultrathin films. The films were deposited at $250{ }^{\circ} \mathrm{C}$. This is lower than the reported temperature of $\mathrm{Mg}$ diffusion in $\mathrm{Fe}_{3} \mathrm{O}_{4}{ }^{12}$ The film thickness dependence of magnetization

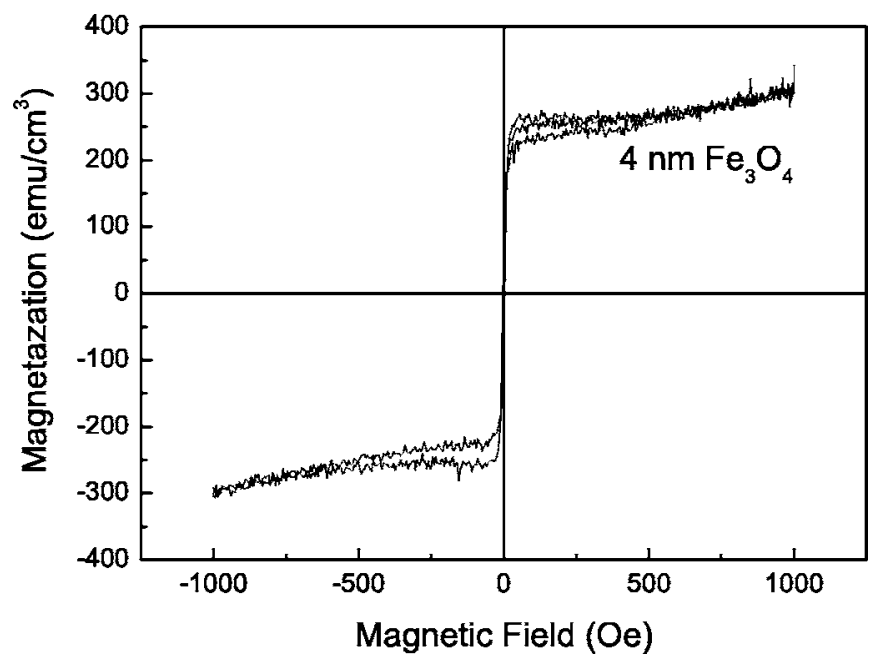

FIG. 4. The hysteresis loop of the $4 \mathrm{~nm}$ thick magnetite film. 


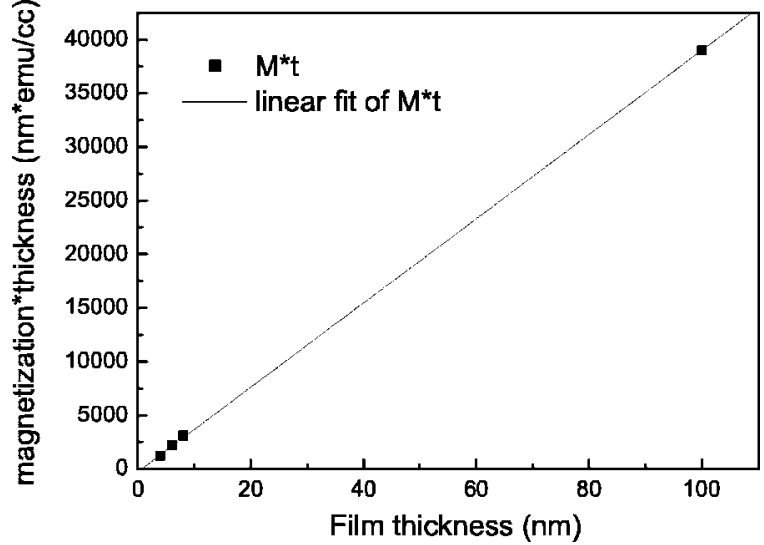

FIG. 5. Film thickness dependence of magnetization multiplied by thickness.

multiplied by the thickness is shown in Fig. 5. We fitted the magnetization by a linear fit. The positive $x$-axis interception of $0.42 \mathrm{~nm}$ is the dead layer, which is about half unit cell of $\mathrm{Fe}_{3} \mathrm{O}_{4}(0.84 \mathrm{~nm})$. This layer is magnetically inactive. The resistivity of the films increases with decreasing film thickness and is generally enhanced compared to the resistivity of the thick films. This might indicate strong scattering of antiphase boundaries and influence of dead layer.

The dependence of magnetoresistance on the thickness of the magnetite films at $120 \mathrm{~K}$ is shown in Fig. 6. The field was applied in the film plane without any specific choice of azimuth. MR depends linearly on the applied magnetic field and MR value increases with the increasing film thickness. MR value also increases with decreasing temperature. MR of magnetite film is related to electron transfer across the APBs. ${ }^{13}$ When a magnetic field is applied, the magnetization of domains separated by the APBs turns towards the external

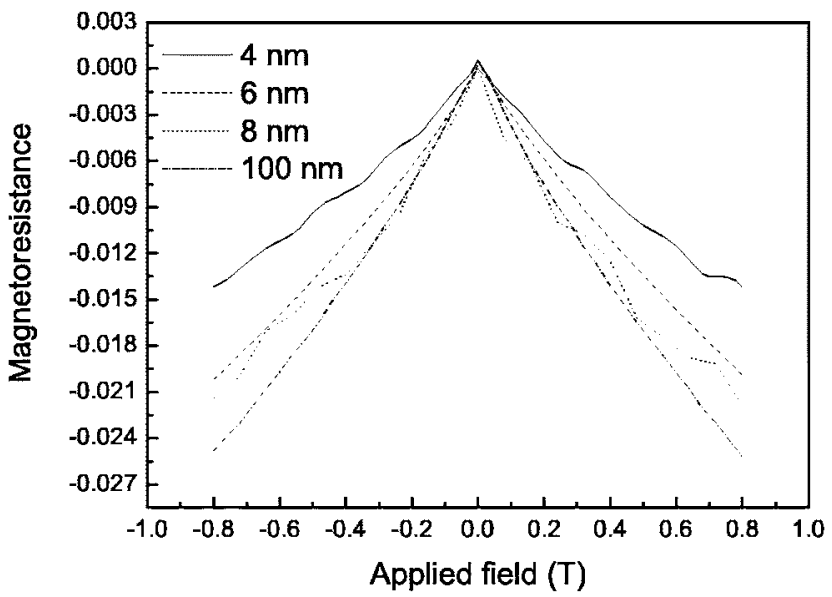

FIG. 6. Dependence of magnetoresistance on thickness of magnetite films at $120 \mathrm{~K}$. field direction and angles between adjacent spins $(\theta)$ decrease, thus, the electrons undertake less scattering between domains. This is the origin of negative MR in magnetite. This is also the basis of greater MR in magnetite films compared to that of bulk. Greater density of APBs is expected in thinner films. We expected to find greater MR value in the thinner films. The results were contrary to the expectation. This could be explained by the existence of "dead layer." The dead layer is magnetic inactive and may reduce the spin polarization of mobile electrons in the entire film. This could result from spin-independent scattering at the dead layer. The effect of dead layer in thinner film is relatively greater than the one in the thicker film and will lead to a lower MR.

\section{CONCLUSIONS}

Ultrathin $\mathrm{Fe}_{3} \mathrm{O}_{4}$ films grown on $\mathrm{MgO}$ (100) single crystal substrates by MBE in the thickness range of 2-8 nm are epitaxial. AFM and $V-I$ curve results confirmed that the films are continuous. The resistivities of the films with temperature results imply that the conductivity mechanism in these films is the same. The films show ferrimagnetic behavior. The dead layer formed by $\mathrm{Mg}$ diffusion between $\mathrm{MgO}$ substrate and magnetite films and also dead layer on the top uncapped film are considered as possible reasons for the anomalies in resistivity and magnetic properties of the ultrathin films. MR depends linearly on the applied magnetic field and MR value increases with the increasing film thickness. The ferrimagnetic ultrathin film with very low coercivity has the potential application in sensors and hard disk drives.

\section{ACKNOWLEDGMENT}

This work was supported by Science Foundation Ireland (SFI) under the Contract No. 00/PI.1/C042.

${ }^{1}$ R. A. de Groot and K. H. J. Buschow, J. Magn. Magn. Mater. 54-57, 1377 (1986).

${ }^{2}$ M. Penicaud, B. Siberchicot, C. B. Sommers, and J. Kubler, J. Magn. Magn. Mater. 103, 212 (1992).

${ }^{3}$ D. T. Margulies, F. T. Parker, M. L. Rudee, F. E. Spada, J. N. Chapman, P. R. Aitchison, and A. E. Berkowitz, Phys. Rev. Lett. 79, 5162 (1997).

${ }^{4}$ F. C. Voogt and T. T. M. Palstra, Phys. Rev. B 57, 8109R (1998).

${ }^{5}$ W. Eerenstein, T. T. M. Palstra, S. S. Saxena, and T. Hibma, Phys. Rev. Lett. 88, 247204 (2002).

${ }^{6}$ Y. Zhou, X. Jin, and I. V. Shvets, J. Appl. Phys. 95, 7357 (2004).

${ }^{7}$ X. W. Li, G. Q. A. Gupta, and G. Xiao, J. Appl. Phys. 83, 734 (1998).

${ }^{8}$ N. F. Mott, Metal-Insulator Transitions, 2nd ed. (Taylor \& Francis, London, 1990), p. 215.

${ }^{9}$ J. Garcia et al., Phys. Rev. B 63, 054110 (2001).

${ }^{10}$ J. S.-Y. Feng, R. D. Pashley, and M.-A. Nicolet, J. Phys. C 8, 1010 (1975).

${ }^{11}$ Y. Zhou, C. McEvoy, and I. V. Shvets, J. Magn. Magn. Mater. 290-291, 1033 (2005).

${ }^{12}$ W. Eerenstein, T. T. M. Palstra, S. S. Saxena, and T. Hibma, Phys. Rev. Lett. 88, 247204 (2002).

${ }^{13}$ M. Ziese and H. J. Blythe, J. Phys.: Condens. Matter 12, 13 (2000). 